\title{
Correction to: A review on preparation and characterization of silver/ nickel oxide nanostructures and their applications
}

\author{
Eman Absi ${ }^{1,2} \cdot$ Muneer Aziz Saleh ${ }^{3} \cdot$ Naif Mohammed Al-Hada ${ }^{4,3,5} \cdot$ Khaidzir Hamzah $^{3}$. \\ Abdulsalam M. Alhawsawi ${ }^{6,7}$. Essam M. Banoqitah ${ }^{8}$
}

Published online: 24 November 2021

(c) The Author(s), under exclusive licence to Springer-Verlag GmbH, DE part of Springer Nature 2021

\section{Correction to: Applied Physics A (2021) 127:871 https://doi.org/10.1007/s00339-021-04979-8}

In this article the following reference was incorrect. Correct reference is as follows.

Adiba, V. Pandey, S. Munjal, T. Ahmad, Structural, morphological and magnetic properties of antiferromagnetic nickel oxide nanoparticles synthesized via sol-gel route, Mater. Today Proc. 26, 3116-3118 (2020). https://doi.org/ 10.1016/j.matpr.2020.02.643

Publisher's Note Springer Nature remains neutral with regard to jurisdictional claims in published maps and institutional affiliations.

The original article can be found online at https://doi.org/10.1007/ s00339-021-04979-8.

Naif Mohammed Al-Hada

naifalhada@yahoo.com

1 Department of Physics, Faculty of Science, Universiti Teknologi Malaysia, Johor Bahru 81310, Malaysia

2 Department of Coastal Environment, School of Basic and Marine Sciences, The University of Jordan-Aqaba Branch, Aqaba, Jordan

3 Nuclear Energy Programme, Faculty of Engineering, School of Chemical and Energy Engineering, Universiti Teknologi Malaysia, Johor Bahru 81310, Malaysia

4 Shandong Key Laboratory of Biophysics, Institute of Biophysics, Dezhou University, Dezhou 253023, China
5 Department of Physics, Faculty of Applied Science, Thamar University, 87246 Dhamar, Yemen

6 Department of Nuclear Engineering, Faculty of Engineering, King Abdulaziz University, P.O. Box 80204, Jeddah 21589, Saudi Arabia

7 Center for Training and Radiation Prevention, King Abdulaziz University, P.O. Box 80204, Jeddah 21589, Saudi Arabia

8 Department of Nuclear Engineering, Faculty of Engineering, K. A. CARE Energy Research and Innovation Center, King Abdulaziz University, P.O. Box 80204, Jeddah 21589, Saudi Arabia 